\title{
Study on IPTV Business Flow Evaluation Model Based on FTTx Network
}

\author{
Ningning Chen \\ Department of Technology, Xi'an International University, Shaanxi Xi'an 710077, China
}

Keywords: IPTV; traffic assessment; business release; FTTx

\begin{abstract}
Accurate flow assessment is the primary issue for operators to distribute new IPTV services and expand their users. This paper mainly conducts research and analysis of IPTV distribution and business expansion brought about by the operation and maintenance of pain points, with FTTx network as the research object, and focuses on the design of an IPTV traffic evaluation model (including IPTV model, IPTV issued new business traffic flow prediction model, user expansion IPTV business planning schedule table). Through the establishment of the model, the IPTV traffic assessment can be completed quickly, and it can help the telecom engineers to make decision on operation and maintenance.
\end{abstract}

\section{Introduction}

IPTV (Internet Protocol Television) is an infrastructure using WBN (wide-band networks). It is a brand-new technology with home TV as the terminal equipment integrating the techniques of internet, multimedia and communication and providing the household users with various interactive digital media services including digital TV. According to the statics of Ministry of Industry and Information Technology of the People's Republic of China, from January to March, 2015, three basic telecommunication enterprises had a net increase of 1.372 million access users of internet broadband, totaling 204 million users, and 2.666 million IPTV users, totaling 36.302 users. As an emerging business, development speed of IPTV has far exceeded that of internet broadband business. In 2016, operation license for broadband network was granted to Broadcast Television Network, and China Mobile obtained the operation license for IPTV business and the policy dividends with the vigorous support of the government for IPTV business. It is preliminarily estimated by Ministry of Industry and Information Technology that IPTV users in 2016 would be doubled. In terms of development speed and development space, the emerging IPTV business far exceeds the business of internet broadband. The rapid development of IPTV business brings about difficulties for the operation, maintenance and planning of telecommunication network, of which, the first question is traffic evaluation ${ }^{[2]}$ when operator issues new IPTV business and expands user scales.

\section{Analysis on Comparative Characteristics of IPTV Business Traffic and Broadband Internet Business Traffic}

According to the typical FTTx networking scenario and user business information: number of broadband users accessing to OLT equipment: 2000; peak online rate: $60 \%$; mean traffic: $400 \mathrm{~Kb} / \mathrm{s}$; number of IPTV channels: 10; IPTV user permeability: 60\%; IPTV peak online rate: 50\%; IPTV live rate: $50 \%$ (click rate: $50 \%$ ), IPTV bandwidth of program: $10 \mathrm{Mb} / \mathrm{s}$ (all are standard programs), it is calculated as the formula below:

Broadband internet traffic $=$ number of bandwidth users * peak online rate of bandwidth $*$ mean traffic $=480 \mathrm{Mb} / \mathrm{s}$

IPTV business traffic $=$ channel number*IPTV bandwidth of program + number of broadband users

* IPTV permeability * IPTV online rate * click rate * IPTV bandwidth of program= 3100Mb/s (2)

Thus, it can be seen that IPTV business traffic is far greater than that of bandwidth online business, and the issuance of new IPTV business and business scale expansion are prone to cause a 
bottleneck for bandwidth. Therefore, the operator is faced with the difficulties currently in two aspects below:

(1) Scenario of new IPTV business issuance: whether current network bandwidth can meet the demand of new IPTV for traffic.

(2) Scenario of IPTV user scale expansion: whether there is a bottleneck in current network bandwidth and whether it can support the operator in the future business planning of IPTV permeability.

Whether network bandwidth can meet the business demand for traffic is the most significant KPI (Key Technical Indicator) when issuing IPTV business and expanding business sale, while, the operator can only check the macro operation state of website through NMS. There isn't a precise traffic evaluation strategy, and telecommunication engineer mainly depends on the physical bandwidth of equipment and user number of the equipment to estimate the business traffic $^{[3]}$ coarsely. However, there isn't detailed data support for such ambiguous evaluation, hence, deviation may occur inevitably, which may cause a bottle neck or redundancy for network bandwidth and bring about difficulties for network maintenance. Therefore, the key problem for establishing a favorable traffic evaluation is that operator must carry forward new IPTV business issuance and user scale expansion. In this paper, it mainly carries out abstract modeling via IPTV business traffic information of equipment and calculates IPTV business traffic demand with existing network collecting data as the input, thus outputs quantized evaluation report to give support to IPTV business issuance and IPTV business scale expansion, as shown in Figure 1 and Figure 2.

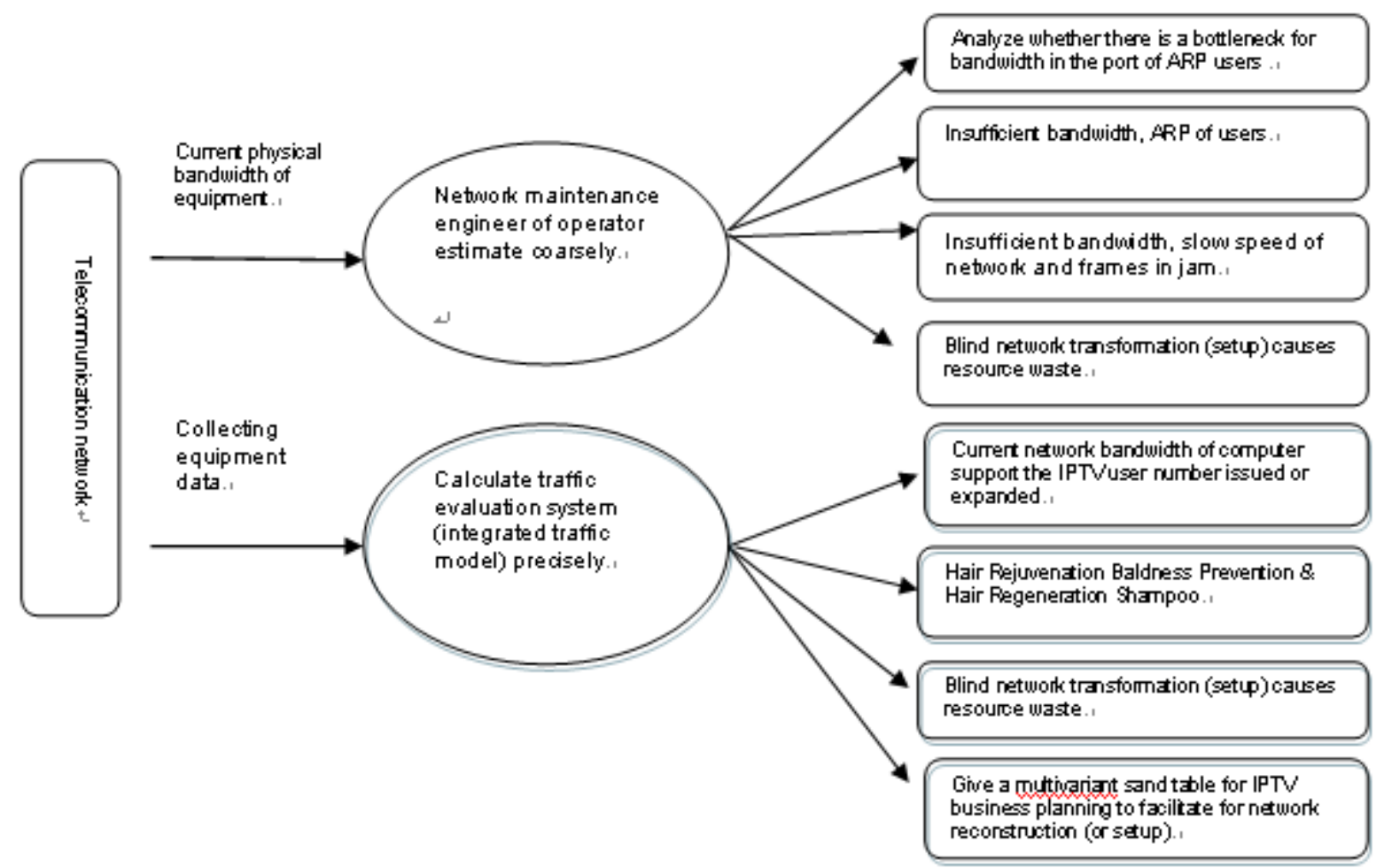

Figure 1 Analysis on Problem Domain and Solutions

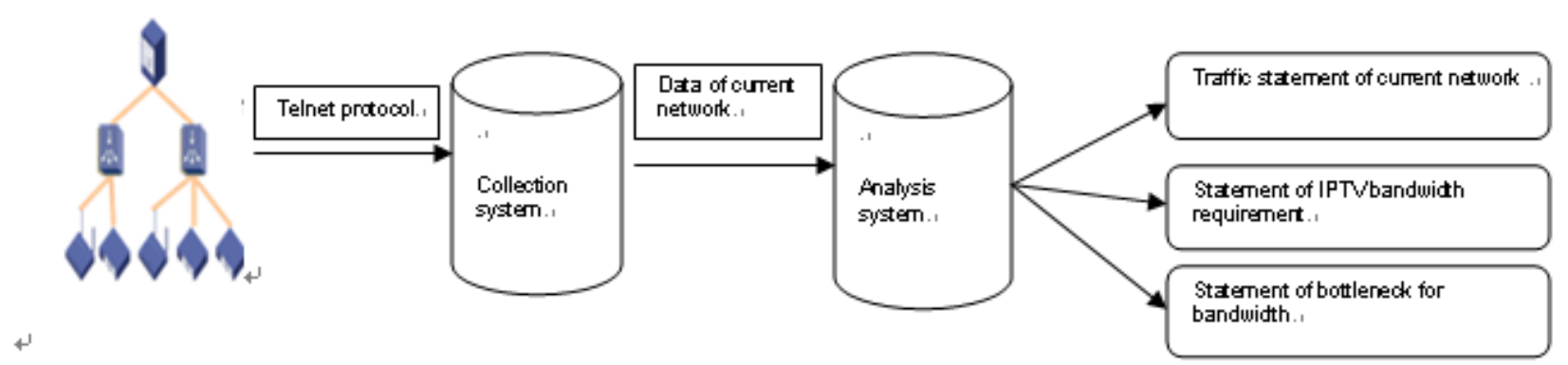

Figure 2 Contents of the System 


\section{Establishment of IPTV Traffic Model}

This paper mainly targets at the maintenance pain point bringing about by new IPTV business issuance and rapid user scale expansion. It is based on FTTx network as study object to complete the design of telecommunication network data collection scheme and traffic modeling, which is mainly composed of four aspects below:

Design telecommunication network data collection scheme, collect FTTx network data via Telnet and SNMP protocol and provide data basis for IPTV traffic evaluation. The data collected mainly includes: relational data of OLT-ONU networking, relevant data of online user number, information data of port traffic (requiring rolling collection), configuration data of NE IPTV multicast $^{[4]}$.

While at the scenario of new IPTV business issuance, IPTV traffic evaluation system must give support to evaluate the traffic information of targeted broadband network, gives out the online user number of each NE port peak value and bandwidth availability of peak value and the answers whether it supports IPTV business issuance, whether there is a bottleneck for bandwidth after issuing IPTV business, what is the detailed data of bottleneck for bandwidth in order that telecommunication engineer can rectify the business based on detailed situation, thus providing guarantee for new IPTV business issuance. Hence, in order to establish new IPTV business issuance traffic model, it is only needed to calculate total traffic required by IPTV business and judge whether current network can satisfy the number allocation demand of IPTV business and give out specific bandwidth of capacity expansion if necessary by collecting data and IPTV business planning information of the operator (mainly for HD \& SD network, location of IPTV multicast replication origin, IPTV business permeability), as shown in Figure 3.

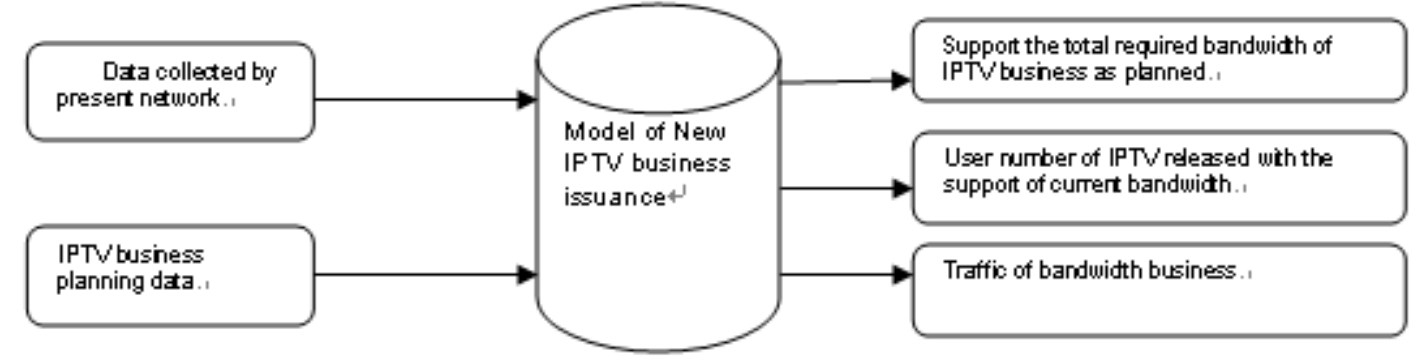

Figure 3 Model of New IPTV Business Issuance

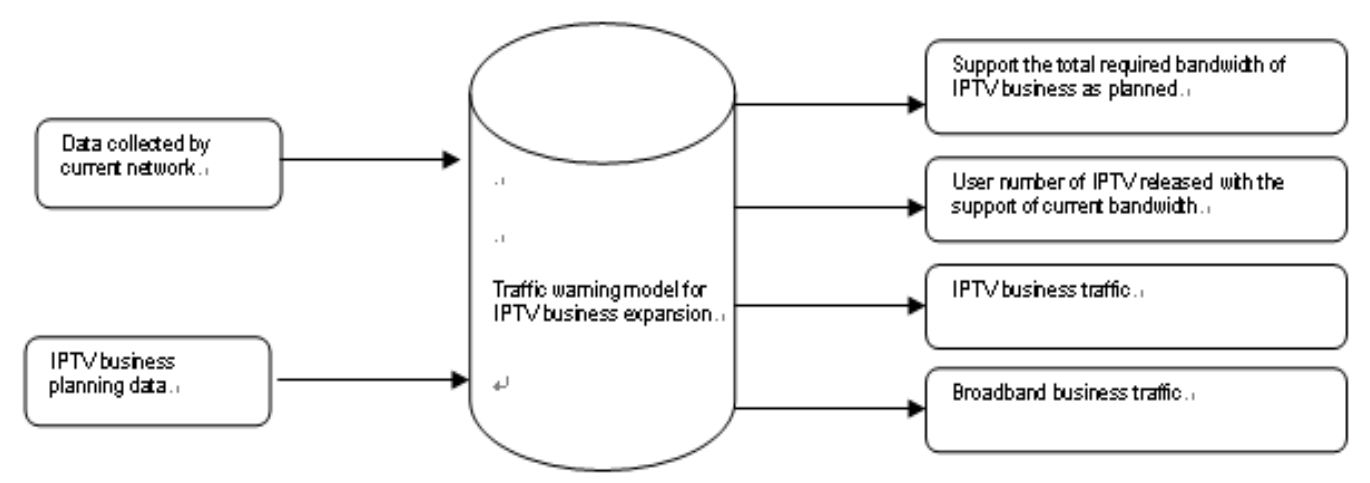

Figure 4 Traffic warning model for IPTV user scale expansion

With the increase of users accessing to network and the rising demands of users for traffic, some network would gradually come across bottleneck for bandwidth causing the connected drop of some users due to the resource competition. This not only exerts strong impact on user experience, but also high complaint rate can make the operation cost of operators increased greatly, even cause user loss. Through traffic evaluation on current network, especially the user network with high complaint rate, it can help positioning the traffic bottleneck point in time and remind operators to transform the business or network. To set up traffic warning model for IPTV user scale expansion, it shall mainly rely on cyclic data collection to analyze the traffic increasing rate of IPTV business 
and use rate of physical bandwidth in NE port and statistic the user network with traffic bottleneck or those will come across traffic bottleneck, as shown in Figure 4.

An excellent network bandwidth planning can support three to five years' development of teleservice in the future. IPTV traffic evaluation can show an increasing trend of user demand for bandwidth through traffic monitoring ${ }^{[5]}$ for users and NE. By combining the operators' positioning on market share and business permeability, it can deduce the number allocation sandboxie in the future, and provide a data base for operators' business planning and network transformation. To set up number allocation sandboxie for IPTV business planning, it shall mainly take IPTV traffic evaluation model (Two scenarios of new business issuance or business scale expansion shall use their respective traffic model algorithm respectively) as the algorithm basis, and the user increase rate, IPTV permeability, HD and SD business, position of multicast replication origin planned by operators as variable parameter, to calculate the network bandwidth demands within designated time in the future, so at to form a multi-scheme deducing sandboxed.

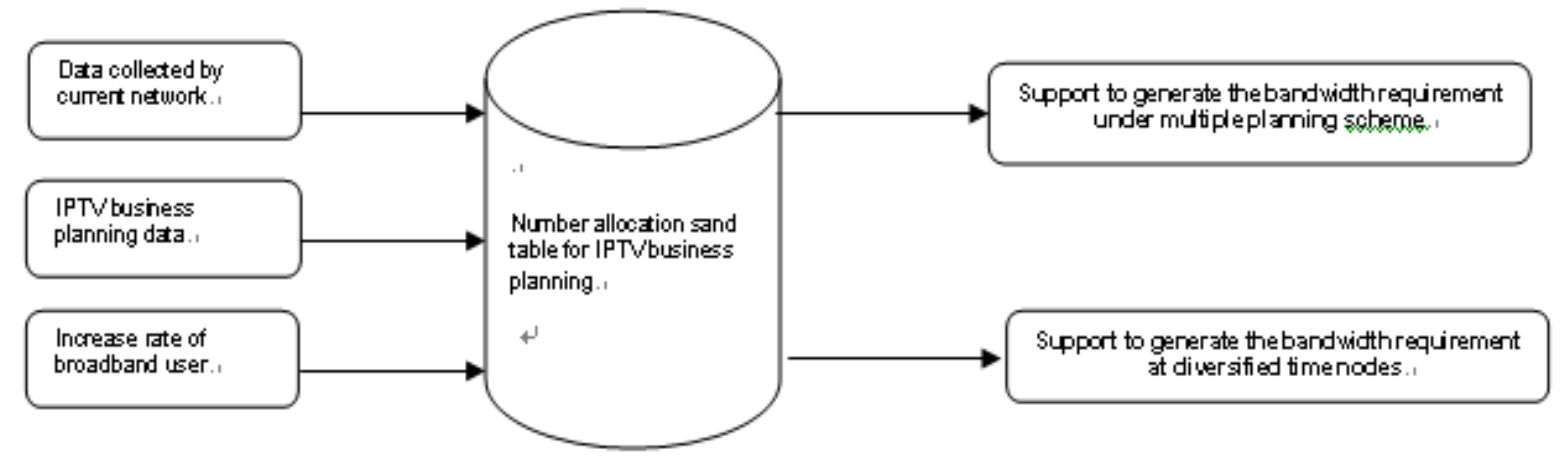

Figure 5 Number allocation sandboxie for IPTV business

\section{Conclusion}

In this paper, it mainly analyzes the status of IPTV in China and the comparative characteristics of IPTV business traffic and bandwidth online traffic. It is summarized that, the operator is faced with the difficulties in two aspects: scenario of new IPTV business issuance and scenario of IPTV user scale expansion. Targeting that traffic evaluation is the primary problem for the operator when carrying forward new IPTV business issuance and user scale expansion, it emphasizes on designing and setting of IPTV traffic evaluation model (including traffic model of new IPTV business issuance, traffic warning model for IPTV user scale expansion, number allocation sandboxie for IPTV business planning). Setting up of the model can help complete IPTV traffic evaluation fleetly, and further assist telecommunication engineer in the decision making of operation and maintenance.

\section{Acknowledgment}

Fund Project:

(1)The Department of science and technology project of Shaanxi provincial in 2016 (Project Number: 2016GY-114)

(2)The social science planning fund project of Xi'an in 2016(Project Number: 16S73)

(3)The social science planning fund project of Xi'an in 2017(Project Number: 17IN09)

\section{References}

[1] Zhang Qian. Research on the development of IPTV industry in Internet audio-visual media -- a case study of IPTV industry in Zhejiang province [D].Zhejiang: Zhejiang Media College, 2016

[2] Wang Hongbo, Cheng Shiduan. Evolution of IPTV Technology [J], China Communications, 2009(2), 47-50

[3] Li Zhenguo. IPTV Technology and Application [J], Radio Engineering. 2007 (12), 53-55 
[4] Zhang Zhibin, Cui Xiaofei. Key technology analysis of IPTV content security [J]. Journal of Shenzhen Institute of Information Technology 2007 (02), 36-40

[5] Qiang Mingjun. Research and application of IPTV network [D]. Hubei: central China normal university, 2007 